\title{
Article \\ Stress Echocardiography Protocol for Deciding Type of Surgery in Ischemic Mitral Regurgitation: Predictors of Mitral Regurgitation Recurrence following CABG Alone
}

\author{
Radoslaw Piatkowski *, Janusz Kochanowski, Monika Budnik, Michal Peller, Marcin Grabowski \\ and Grzegorz Opolski
}

check for

updates

Citation: Piatkowski, R.; Kochanowski, J.; Budnik, M.; Peller, M.; Grabowski, M.; Opolski, G. Stress Echocardiography Protocol for Deciding Type of Surgery in Ischemic Mitral Regurgitation: Predictors of Mitral Regurgitation Recurrence following CABG Alone. J. Clin. Med. 2021, 10, 4816. https://doi.org/ $10.3390 / \mathrm{jcm} 10214816$

Academic Editor: Kana Fujikura

Received: 31 July 2021

Accepted: 19 October 2021

Published: 20 October 2021

Publisher's Note: MDPI stays neutral with regard to jurisdictional claims in published maps and institutional affiliations.

Copyright: (c) 2021 by the authors. Licensee MDPI, Basel, Switzerland. This article is an open access article distributed under the terms and conditions of the Creative Commons Attribution (CC BY) license (https:/ / creativecommons.org/licenses/by/ $4.0 /)$. 1st Chair and Department of Cardiology, Medical University of Warsaw, 02-097 Warsaw, Poland; kochanowski.janusz@gmail.com (J.K.); moni.budnik@gmail.com (M.B.); michalpeller@gmail.com (M.P.); radekp1@wp.pl (M.G.); radekp1@mp.pl (G.O.)

* Correspondence: radekp1@gmail.com; Tel.: +48-22-599-2958

\begin{abstract}
Purpose: Although coronary artery bypass grafting alone (CABGa), or, with mitral annuloplasty (CABGmp), is considered the best therapeutic strategy for patients with ischemic mitral regurgitation (IMR), some recurrences are still reported. The aim of this study was to evaluate the use of the mitral deformation indices (MDI) as a predictor of recurrence of mitral regurgitation in a 12-month follow-up after CABG alone. Methods: A total of 145 patients after myocardial infarction with significant IMR, eligible for CABG, were prospectively enrolled in the study. Mitral valve morphology, left ventricle function, IMR degree as assessed by effective regurgitation orifice area (ERO), myocardial viability, and MDI were assessed prior to surgery. Patients were referred for CABGa (gr.1; $n=90$ ) or CABGmp (gr.2; $n=55$ ) based on clinical assessment, and the results of rest and stress echocardiography (exercise echocardiography and low dose dobutamine echocardiography-DBX). One year after surgery, each patient underwent the evaluation of cardiovascular events. Univariable logistic regression analysis was used to identify the factors of recurrence of IMR in 1 year follow-up. Serial echo examinations were performed in all patients at discharge, and at 1 and 12 months after surgery. Results: Logistic regression analysis revealed that in $\mathrm{CABGa}$, group preoperative changes of tenting area (TA) and coaptation high $(\mathrm{CH})$ during DBX remained the predictors of the recurrence of IMR in 12 months follow-up. TAdbx $>1 \mathrm{~cm}^{2}$ provided a sensitivity of $90 \%$ and specificity of $29 \%$, (AUC 0.6436). The best cut-off value for CHdbx was $0.4 \mathrm{~cm}$ (sensitivity $90 \%$, specificity $34 \%$; AUC 0.6432). In both groups (CABGa vs. CABGmp) no significant differences were observed in 12-month mortality $(1.2 \%$ vs. $0 \% ; p=1.0)$, hospitalizations due to the heart failure (HF) exacerbation $(5.9 \%$ vs. $8.5 \% ; p=0.72)$, and in the incidence of the composite endpoint (deaths/CV hosp/stroke) (7\% vs. $8.5 \% ; p=0.742$ ). Conclusions: The preoperative assessment of MDI changes during dbx can be used to identify patients with IMR qualified to CABG alone at increased risk of recurrence of IMR in 1 year follow-up. Mitral deformation analysis should be used for a better qualification of patients with IMR to the exact surgical approach.
\end{abstract}

Keywords: stress echo; ischemic mitral regurgitation; tenting area; coaptation high

\section{Introduction}

Ischemic mitral regurgitation (IMR) worsens the prognosis of patients after coronary artery disease, both in the short-term and in the long-term follow-up [1-3]. IMR is a dynamic process, and its severity changes with hemodynamic variation. IMR is predominantly related to left ventricle (LV) remodeling and mitral valve deformation [4-7]. LV spherization, mitral annulus enlargement, increased tenting area (TA) and volume, and the loss of systolic mitral annular contraction all contribute to the development of IMR [8]. The treatment for IMR is still a heavily debated topic with an absence of definitive evidence to support either school of thought. The complex pathomechanism of IMR development 
is responsible for problems when elaborating efficient therapeutic methods in patients qualified to CABG alone. Recently, due to better understanding of this complex disease and better medical management options for coronary artery disease, the results of surgical treatment have improved. These surgical options include an isolated CABG procedure to ensure revascularization or mitral valve replacement along with CABG as combined procedure or other mitral valve repair techniques with CABG. Restrictive annuloplasty combined with $\mathrm{CABG}$ is the most often performed surgical procedure to treat patients with significant IMR. However, the sobering results of the current strategies create the need for a better preoperative assessment of the mitral valve and LV geometry and function $[9,10]$. A strict correlation between stress-induced (exercise and dobutamine) changes of IMR, mitral deformation indexes (MDI), myocardium viability, clinical symptoms, and prognosis should be considered when evaluating the eligibility of patients with moderate or severe IMR for the appropriate type of surgery [11-14]. This would help improve risk stratification and the identification of the subgroups of patients with risk of recurrent IMR and worse prognosis who could likely benefit from different surgical strategies. In our opinion, the greater severity of stress induced IMR and changes of MDI correlates with a greater necessity to perform mitral valve repair to avoid the risk of the recurrence of IMR in long-term follow-up.

The aim of this study was to assess the significance of echocardiographic parameters achieved from stress echo (exercise echocardiography and low dose DBX) as a predictor of recurrent IMR in patients with moderate or severe IMR qualified to CABG alone in 12-month follow-up.

\section{Materials and Methods}

\subsection{Study Population}

This prospective observational cohort study included patients aged $>18$, with a history of myocardial infarction and eligible for CABG [15]. All patients had moderate or severe IMR caused by restrictive systolic leaflet motion (Carpentier's type IIIb) with or without annular dilatation (Carpentier's type I). The study was conducted between 2010 and 2017.

A total of 170 potentially eligible patients with IMR qualified to CABG were enrolled in the present study. Of these, 25 patients were excluded from the analysis ( 7 patients had a poor acoustic window for dobutamine echocardiography; 4 patients in whom viability was not demonstrated within left ventricular segments with impaired contractility; 14 had contraindications for exercise). Finally, 145 patients were qualified to CABGa or CABGmp and prospectively enrolled into the study. (Figure 1). The time frame for enrollment of patients into the study was 24 months.

The patients were divided into groups according to the type of intervention planned: group I ( $n=90)$ - CABG alone (CABGa); group II $(n=55)$ —CABG combined with mitral annuloplasty (CABGmp). Additionally, 9 patients were qualified for CABG with mitral valve replacement (CABGmr), but this group was excluded from the analysis. Based on exercise echocardiography (ExE) and dobutamine stress echocardiography (DBX), a diagnostic algorithm for a precise determination of the range of surgical intervention has been elaborated (Table 1). Patients had to meet all criterion to be placed in a given category. Patients were qualified to CABGa if IMR deformation parameters improved during preoperative DBX and were not impaired during EXE (EROexe $<20 \mathrm{~mm}^{2}$ ). The eligibility of patients with an increase in IMR with exercise for CABGa or CABGmp was determined by the results of DBX. The improvement of mitral valve deformation parameters with DBX was the main determinant of eligibility for the CABGa group. On the other hand, CABGmp was considered for patients without an improvement in mitral valve geometry during DBX (Table 1). 


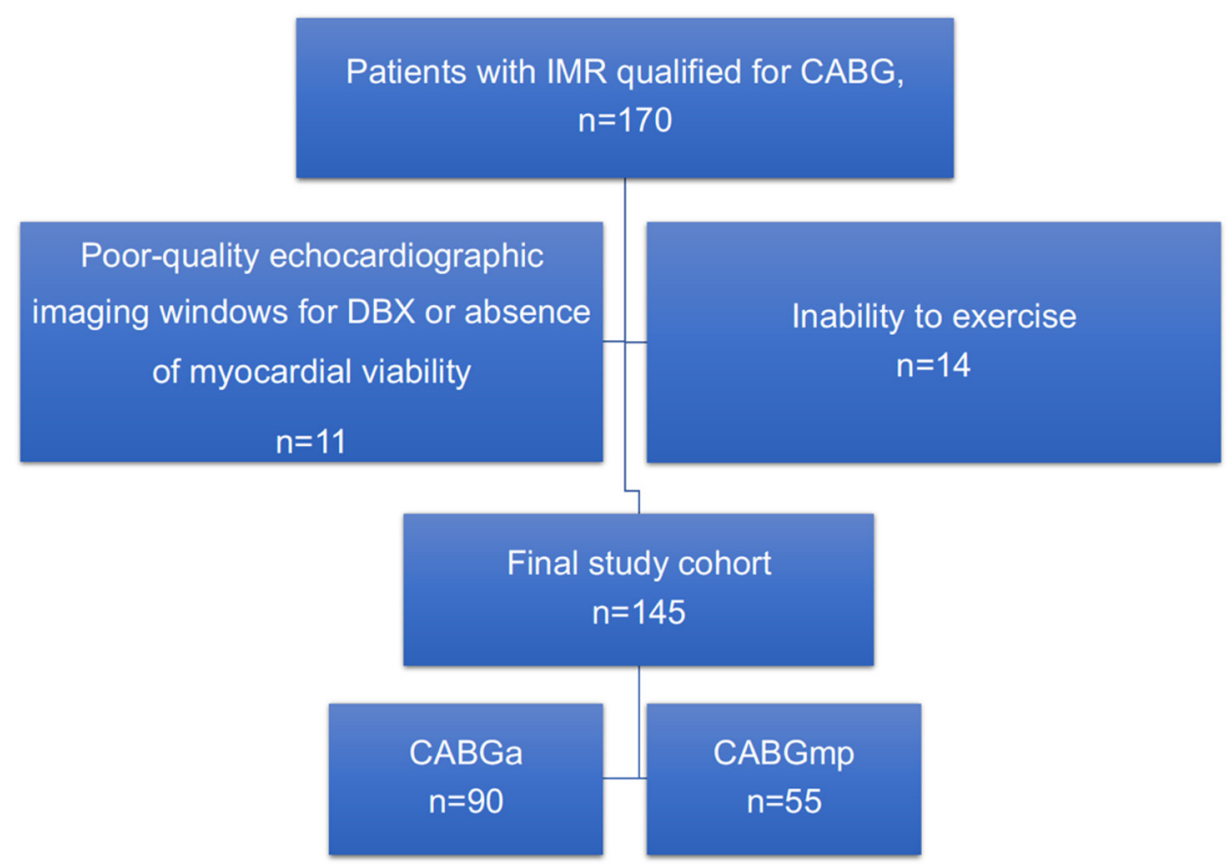

Figure 1. Study flowchart. CABG—-coronary artery bypass grafting; DBX-dobutamine stress echocardiography.

Table 1. Eligibility criteria to a given type of surgery.

\begin{tabular}{cccc}
\hline & CABGa & CABGmp & CABGmr (Not Included in Analysis) \\
\hline ERO exe & $<20 \mathrm{~mm}^{2}$ or $\geq 20 \mathrm{~mm}^{2}$ & $\geq 20 \mathrm{~mm}^{2}$ & $\geq 20 \mathrm{~mm}^{2}$ \\
\hline $\mathrm{CH} \mathrm{dbx}$ & $\leq 6 \mathrm{~mm}$ & $6 \mathrm{~mm}<\mathrm{CH} \leq 10 \mathrm{~mm}$ & $\mathrm{CH}>10 \mathrm{~mm}$ \\
\hline TA dbx & $\leq 1.2 \mathrm{~cm}^{2}$ & $1.2 \mathrm{~cm}^{2}<\mathrm{TA} \leq 2.5 \mathrm{~cm}^{2}$ & $\mathrm{TA}>2.5 \mathrm{~cm}^{2}$ \\
\hline ERO dbx & $<10 \mathrm{~mm}^{2}$ & $-*$ & $-^{*}$ \\
\hline
\end{tabular}

exe-exercise echocardiography; dbx-dobutamine echocardiography; CABGa-coronary artery bypass grafting alone; CABGmp - coronary artery bypass grafting with mitral annuloplasty; CABGmr-coronary artery bypass grafting with mitral replacement; WMSI-wall motion score index; ERO-effective regurgitant orifice area; TA — tenting area; $\mathrm{CH}$ - coaptation height; *-insignificant in qualification strategy.

The study inclusion criterion was the presence of a significant area of viable myocardium found during DBX (improvement in wall motion of at least four dysfunctional segments). The assessment and presence of myocardial viability was necessary for inclusion in both study groups. The exclusion criteria included a left bundle branch block (LBBB), unstable angina, prosthetic heart valve, other valvular or congenital heart diseases, history of CABG, and severe heart failure (HF) symptoms (NYHA IV—New York Heart Association).

Only patients who underwent complete revascularization were included in the further analysis.

An echocardiographic and clinical assessment was performed at discharge, after 1 month and 12 months.

Each patient signed an informed consent form, and the study was approved by the institutional review board of the Medical University of Warsaw. The study was conducted according to the principles stated in the Declaration of Helsinki.

\subsection{Surgery}

The same surgical team performed surgery through a median sternotomy. Regardless of the proposed way of treatment, the final decision on the surgical technique to be used within the mitral valve was always made by the operating surgeon. However, this did not result in any change in the original treatment group assignment. In all patients, CABG was performed using cardiopulmonary bypass in moderate hypothermia with crystalloid 
and blood cardioplegia. The main aim of the surgery was to perform a complete coronary revascularization and, in some patients, mitral restrictive annuloplasty. The ring size was determined after measuring of the height of the anterior leaflet and intertrigonal distance, and then downsizing by two sizes (undersizing annuloplasty) [16,17].

The intraoperative criteria for a successful surgery were as follows: leaflet coaptation height $(\mathrm{CH}) \leq 0.6 \mathrm{~cm}$, tenting area $(\mathrm{TA}) \leq 1.2 \mathrm{~cm}^{2}$, and IMR $\leq 1$ grade. Recurrent IMR was the insufficiency of at least moderate $\left(\mathrm{ERO}>10 \mathrm{~mm}^{2}\right)$ or more at follow-up visits.

\subsection{Echocardiography}

Transthoracic echocardiograms (TTE) were performed within 2-3 days before surgery, and serial TTE examinations were performed at discharge and at follow-up visits. All measurements were performed using the iE33 system (version 4.2-5.0; Philips Medical Systems, N.A., Bothell, WA, USA) equipped with a broadband transducer for a TTE of 2.5-3.5 MHz frequency. IMR severity was assessed by measuring the effective regurgitant orifice area (ERO), with ERO $>10 \mathrm{~mm}^{2}$ and $<20 \mathrm{~mm}^{2}$ considered moderate and ERO $\geq 20 \mathrm{~mm}^{2}$ considered severe, as well as mitral regurgitation volume (MRvol) with $\mathrm{MR}$ vol $\geq 30 \mathrm{~mL}$ considered severe [18-20]. ERO and MRvol were calculated using flow convergence (proximal isovelocity surface area-PISA method). The radius of the PISA (r) was measured from the vena contracta level to the point of color Doppler aliasing. ERO was calculated as: $6.28 \times \mathrm{r}^{2} \times \mathrm{Va} /$ Peak V RegJet, where Va is aliasing velocity and VRegJet is the peak velocity of the regurgitant jet by Continuous Wave Doppler. The MRvol was calculated as ERO $\times$ VTI RegJet, where VTI RegJet is the VTI of the regurgitant jet [21].

Wall motion abnormalities were evaluated in accordance with the recommendations of the American Society of Cardiology [22]. The wall motion score index (WMSI) was calculated according to a 17-segment model [23]. The left ventricular volumes and ejection fraction (EF) were assessed by the biapical Simpson disk method. The mitral valve deformation was evaluated by measuring the tenting area (TA), i.e., the area enclosed between mitral leaflets and the line of the annular plane and the coaptation height $(\mathrm{CH})$, i.e., the distance between leaflet coaptation and the mitral annular plane from the parasternal long-axis view at mid-systole [24].

\subsection{Stress Echocardiography}

Low-dose DBX was used to distinguish akinetic viable segments from nonviable myocardial regions [25]. The presence of a significant area of viable LV myocardium was the condition for patient inclusion for further analysis. Additionally, during DBX, the dynamics of MDI (increase or decrease) and IMR changes were analyzed. DBX was performed in accordance with current guidelines [26]. For the detection of inotropic response in heart failure patients, we used stages of $5 \mathrm{~min}$, starting from 5 up to $20 \mathrm{mg} / \mathrm{kg} / \mathrm{min}$. The next step of qualification for the surgery included ExE to assess the dynamics of IMR changes and TRPG (tricuspid regurgitation pressure gradient as the exponent of the right ventricle overload).

All subjects also underwent a symptom-limited graded exercise echocardiography (ExE) test to assess the dynamics of IMR changes and TRPG, the latter as the exponent of right ventricle overload. The symptom-limited grade ExE was performed according to the following protocol: the initial workload of 25 watts (WAT) was maintained for $3 \mathrm{~min}$, and then the workload was increased every 2 min by $25 \mathrm{~W}$. Blood pressure and a 12-lead electrocardiogram were recorded every $2 \mathrm{~min}$. Two-dimensional and Doppler echocardiographic recordings were available throughout the test. Exercise was interrupted when ischemic electrocardiographic signs, fatigue, or intolerable dyspnea appeared [26].

\subsection{Clinical End Points}

The efficacy of the diagnostic algorithm was evaluated by analyzing the results obtained during the 12-month follow-up CABGa group of patients, which included:

- functional status (NYHA, CCS-Canadian Cardiovascular Society). 
- dynamics of selected LV parameters changes (EF, WMSI).

- analysis of the recurrence of moderate or severe IMR.

- midterm (12 months) mortality; and

- hospitalization due to exacerbation of HF symptoms.

\subsection{Data Collection}

Baseline clinical characteristics (demographics, medical history, and therapy) and echocardiography (rest, stress) examinations performed at the baseline visit were retrieved from patient's medical records. Preoperative and postoperative clinical status was determined according to the criteria of the NYHA and the CCS functional class for HF and angina, respectively.

\subsection{Statistical Analysis}

All continuous variables presented non-normal distribution based on a Shapiro-Wilk test and were demonstrated as median values and interquartile ranges. Categorical variables were presented as the number of patients and percentages. Differences between groups for categorical and continuous data were analyzed by Fisher's exact test and a Mann-Whitney U test, respectively. To establish the predictive values of analyzed variables, univariate logistic regression models were used. Because of the low value of events per variable, multivariate models were not analyzed. Predicting values of variables were presented as ROC curves. All tests were two-tailed, and a $p$ value of 0.05 or less was considered statistically significant. All analyses were performed using SAS statistical software, version 9.4 (SAS Institute Inc., Cary, NC, USA).

\section{Results}

\subsection{Baseline Characteristics}

Tables 2 and 3 show the preoperative clinical and echocardiographic variables in two analyzed groups (CABGa and CABGmp). The patients in the CABGmp group were in a higher NYHA class and had a higher incidence of chronic kidney disease. As expected, patients in the CABGmp group had lower EF, higher WMSI, larger LA, LV dimensions and volumes, and lower EF than patients in the CABGa group. Compared with the CABGa group, significantly higher values of MDI and systolic mitral area were observed in CABGmp. During ExE, significant differences concerning workload were seen in the given groups of patients. Patients in the CABGmp group showed a significant exercise induced IMR and TRPG increase. During DBX, in the CABGa group, the normalization of MDI and a significant decrease in the IMR value were observed $(p<0.001)$; in CABGmp, moderate/severe IMR persisted. During DBX, in the CABGmp group, no significant improvement in MDI was noticed.

Table 2. Baseline characteristic of patients with significant MR treated by CABGa or CABGmp.

\begin{tabular}{|c|c|c|c|}
\hline & $\begin{array}{c}\text { CABGa } \\
n=90\end{array}$ & $\begin{array}{c}\text { CABGmp } \\
n=55\end{array}$ & $p$ Value \\
\hline Male sex, $n(\%)$ & $50(55.6 \%)$ & $31(56.4 \%)$ & 1.0000 \\
\hline $\mathrm{BMI}\left(\mathrm{kg} / \mathrm{m}^{2}\right)$ & $26.9(17.6-37.5)$ & $26.4(17.8-35.6)$ & 0.4302 \\
\hline Hypertension, $n(\%)$ & $58(64.4 \%)$ & $39(70.9 \%)$ & 0.4703 \\
\hline Diabetes, $n(\%)$ & $27(30 \%)$ & $22(40 \%)$ & 0.2777 \\
\hline Hyperlipidemia, $n(\%)$ & $58(64.4 \%)$ & $26(47.3 \%)$ & 0.0563 \\
\hline Smoking, $n(\%)$ & $61(68 \%)$ & $37(67.3 \%)$ & 0.8558 \\
\hline Family history of CAD, $n(\%)$ & $44(48.9 \%)$ & $24(43.6 \%)$ & 0.6081 \\
\hline
\end{tabular}


Table 2. Cont.

\begin{tabular}{|c|c|c|c|}
\hline & $\begin{array}{c}\text { CABGa } \\
n=90\end{array}$ & $\begin{array}{c}\text { CABGmp } \\
n=55\end{array}$ & $p$ Value \\
\hline Chronic kidney disease, $n(\%)$ & $10(11.1 \%)$ & $14(25.5 \%)$ & 0.0368 \\
\hline Atrial fibrillation, $n(\%)$ & $15(16.7 \%)$ & $16(29.1 \%)$ & 0.0956 \\
\hline COPD, $n(\%)$ & $8(8.9 \%)$ & $7(12.7 \%)$ & 0.5758 \\
\hline NYHA & $1.9(0-4)$ & $2.4(0-4)$ & 0.0032 \\
\hline One vessel CAD, $n(\%)$ & $2(2.2 \%)$ & $10(10.9 \%)$ & 0.0535 \\
\hline Two vessels CAD, $n(\%)$ & $22(24.4 \%)$ & $14(25.5 \%)$ & 1.0000 \\
\hline Three vessels CAD, $n(\%)$ & $66(73 \%)$ & $35(63.6 \%)$ & 0.2664 \\
\hline \multicolumn{4}{|l|}{ Affected vessel treated by CABG, $n(\%)$} \\
\hline LMCA & $21(23.3 \%)$ & $14(25.5 \%)$ & 0.8423 \\
\hline LAD & $89(98.9 \%)$ & $47(85.5 \%)$ & 0.0019 \\
\hline $\mathrm{Cx}$ & $70(77.8 \%)$ & $39(70.9 \%)$ & 0.4288 \\
\hline RCA & $78(86.7 \%)$ & $47(85.5 \%)$ & 1.0000 \\
\hline
\end{tabular}

Data are presented as median values and interquartile ranges, or number (percentage) as shown; CAD—coronary artery disease; BMI—body mass index; COPD—chronic obstructive pulmonary disease; NYHA-New York Heart Association; LMCA-left main coronary artery; LAD-left anterior descending artery; Cx-circumflex artery; RCA—right coronary artery.

Table 3. Baseline echocardiographic parameters in patients with IMR treated by CABGa or CABGmp.

\begin{tabular}{|c|c|c|c|}
\hline & $\begin{array}{c}\text { CABGa } \\
n=90\end{array}$ & $\begin{array}{c}\text { CABGmp } \\
n=55\end{array}$ & $p$ Value \\
\hline \multicolumn{4}{|l|}{ Rest echocardiography } \\
\hline $\mathrm{EF}(\%)$ & $43.6(18-65)$ & $39.7(20-60)$ & 0.0184 \\
\hline WMSI & $1.6(1.1-2.7)$ & $1.7(1.2-2.5)$ & 0.0024 \\
\hline LVDD (mm) & $53.6(43-69)$ & $56.4(44-72)$ & 0.0130 \\
\hline LVDS (mm) & $40.1(26-58)$ & $43.8(30-64)$ & 0.0079 \\
\hline EDV $(m L)$ & $125.6(47-283)$ & $144(66-252)$ & 0.0265 \\
\hline ESV (mL) & $75.1(18-213)$ & $89.8(27-174)$ & 0.0363 \\
\hline $\mathrm{LA}(\mathrm{mm})$ & $41.6(30-58)$ & $44.3(34-54)$ & 0.0022 \\
\hline RV (LAX) (mm) & $25(19-36)$ & $26.8(20-38)$ & 0.0018 \\
\hline SIs & $0.38(0.17-0.58)$ & $0.41(0.16-0.9)$ & 0.1120 \\
\hline SId & $0.49(0.23-0.77)$ & $0.52(0.25-0.94)$ & 0.1993 \\
\hline $\mathrm{VC}(\mathrm{mm})$ & $5.2(4-8)$ & $6.8(1-13)$ & $<0.0001$ \\
\hline PISA (mm) & $6.2(4-8)$ & $7.5(5-11)$ & $<0.0001$ \\
\hline $\mathrm{ERO}\left(\mathrm{mm}^{2}\right)$ & $15.6(11-30)$ & $23.3(11-49)$ & $<0.0001$ \\
\hline MR vol (mL) & $23.9(12-43)$ & $35.9(17-72)$ & $<0.0001$ \\
\hline Coaptation height (mm) & $7(4-14)$ & $10(6-14)$ & $<0.0001$ \\
\hline Tenting area $\left(\mathrm{cm}^{2}\right)$ & $1.9(1.2-3.8)$ & $2.7(1.5-5.7)$ & $<0.0001$ \\
\hline SMA $\left(\mathrm{cm}^{2}\right)$ & $9.8(7.1-13.1)$ & $10.7(7.2-14.8)$ & 0.0008 \\
\hline Dobutamine echocardiography, $n(\%)$ & $90(100 \%)$ & $55(100 \%)$ & \\
\hline $\mathrm{EF}(\%)$ & $51.3(25-77)$ & $45.4(24-64)$ & 0.0010 \\
\hline WMSI & $1.3(1-2.1)$ & $1.5(1-2.3)$ & 0.0004 \\
\hline TRPG (mmHg) & $23.4(0-44)$ & $31.5(12-54)$ & $<0.0001$ \\
\hline
\end{tabular}


Table 3. Cont.

\begin{tabular}{|c|c|c|c|}
\hline & $\begin{array}{c}\text { CABGa } \\
n=90\end{array}$ & $\begin{array}{c}\text { CABGmp } \\
n=55\end{array}$ & $p$ Value \\
\hline PISA (mm) & $3.9(2-7)$ & $6.8(2-10)$ & $<0.0001$ \\
\hline $\mathrm{ERO}\left(\mathrm{mm}^{2}\right)$ & $8(3-16)$ & $19.8(0.06-43)$ & $<0.0001$ \\
\hline MR vol (mL) & $13.2(4-33)$ & $29.7(2-60)$ & $<0.0001$ \\
\hline Tenting area $\left(\mathrm{cm}^{2}\right)$ & $1.2(0.6-3.3)$ & $2.4(1.3-4.8)$ & $<0.0001$ \\
\hline SMA & $8.6(5.8-12)$ & $9.6(6.7-13.9)$ & 0.0003 \\
\hline Exercise echocardiography, $n(\%)$ & $90(100 \%)$ & $55(100 \%)$ & \\
\hline Workload (Watts) & $63.5(25-100)$ & $60.9(25-100)$ & $p=0.434$ \\
\hline $\mathrm{EF}(\%)$ & $45.5(19-65)$ & $38.3(18-64)$ & $<0.0001$ \\
\hline TRPG (mmHg) & $30.0(8-69)$ & $48.2(25-81)$ & $<0.0001$ \\
\hline PISA (mm) & $5.9(2-9)$ & $8.9(7-12)$ & $<0.0001$ \\
\hline $\mathrm{ERO}\left(\mathrm{mm}^{2}\right)$ & $15(4-33)$ & $32.7(19-79)$ & $<0.0001$ \\
\hline MR vol (mL) & $22.9(5-56)$ & $46.8(25-119)$ & $<0.0001$ \\
\hline
\end{tabular}

Data are presented as median values and interquartile ranges. TAPSE-tricuspid annular plane systolic excursion; rest-echo examination at rest; exe-exercise; dbx—dobutamine echocardiography; MR—-mitral regurgitation; WMSI—wall motion score index; VC—vena contracta; PISA — proximal isovelocity surface area; ERO_effective regurgitant orifice; MRvol—mitral regurgitation volume; TA—-tenting area; $\mathrm{CH}$ —coaptation height; SMA — systolic mitral area; EF—ejection fraction; LVDD—left ventricular end-diastolic dimension; LVDS—left ventricular end-systolic dimension; SIs—sphericity index at end-systole; SIs—sphericity index at end-diastole; EDV—left ventricular end-diastolic volumes; ESV—left ventricular end-systolic volume; TRPG—-maximal tricuspid regurgitant peak gradient.

\subsection{In-Hospital Outcomes}

We observed significant differences in the rate of in-hospital serious adverse events between the CABGa group and the combined procedure group (Table 4). There was significantly longer length in hospital stay, higher rate of acute kidney disease, respiratory failure, and heart failure events in the combined-procedure group. There was also a higher rate of in-hospital atrial fibrillation $(30.3 \%$ vs. $51.9 \%, p=0.0132)$ in the combined-procedure group than in the CABGa group. In-hospital mortality was higher in the CABGmp group (CABGa vs. CABGmp: $5.6 \%$ vs. $16.4 \%, p<0.0001$ ).

Table 4. In-hospital complications in patients with IMR treated by CABGa or CABGmp.

\begin{tabular}{cccc}
\hline & CABGa & $n=90$ & CABGmp \\
$n=55$ & $p$ Value & \\
\hline Length of hospitalization (days) & $20.5(4-80)$ & $29.9(2-114)$ & 0.0069 \\
\hline Atrial fibrillation, $n(\%)$ & $27(30.3 \%)$ & 0.0132 & 0.5266 \\
\hline Ventricular tachycardia/Ventricular fibrillation, $n(\%)$ & $2(2.3 \%)$ & $0(0 \%)$ & 0.1409 \\
\hline TIA/stroke, $n(\%)$ & $0(0 \%)$ & $2(3.7 \%)$ & 0.1881 \\
\hline Infection, $n(\%)$ & $22(24.7 \%)$ & $19(35.2 \%)$ & 0.0004 \\
\hline Acute kidney disease on dialysis, $n(\%)$ & $4(4.5 \%)$ & $14(25.9 \%)$ & $<0.0001$ \\
\hline Respiratory failure, $n(\%)$ & $1(1.1 \%)$ & $11(20.4 \%)$ & $<0.0001$ \\
\hline IABP, $n(\%)$ & $11(12.4 \%)$ & $11(20.4 \%)$ & 0.0041 \\
\hline Cardiogenic shock, $n(\%)$ & $4(4.5 \%)$ & $24(44.4 \%)$ & 0.0277 \\
\hline Deed of antiarrhythmic medication use, $n(\%)$ & $23(25.8 \%)$ & $4(7.4 \%)$ & 0.0674 \\
\hline Dleeding, $n(\%)$ & $1(1.1 \%)$ & $9(16.4 \%)$ & 0.0443 \\
\hline
\end{tabular}

Data are presented as median values and interquartile ranges, or number (percentage) as shown; TIA—transient ischemic attack; IABP— intra-aortic balloon pump. 


\subsection{Echocardiographic Results/Recurrence Mitral Regurgitation}

After CABG alone, or with mitral annuloplasty, TTE evaluation revealed acceptable results in all the cases: there was no MR or mild MR. Before discharge, TTE was performed, which confirmed the good results of surgical treatment in both groups of patients. Unfortunately, at 1 year, the prevalence of moderate or severe IMR $\left(E R O>10 \mathrm{~cm}^{2}\right)$ was higher in the CABGa group than in the combined-procedure group $(25 \%$ vs. $17 \%, p<0.01)$ (Table 5$)$.

Table 5. Follow-up after 1-year post surgery.

\begin{tabular}{|c|c|c|c|}
\hline & $\begin{array}{c}\text { CABGa } \\
n=90\end{array}$ & $\underset{n=55}{\text { CABGmp }}$ & $p$ Value \\
\hline $\begin{array}{l}\text { Recurrence of IMR }(\text { ERO }>10 \mathrm{~cm}) n,(\% \\
\text { patients })\end{array}$ & $28(25 \%)$ & $9(17 \%)$ & $p<0.01$ \\
\hline $\begin{array}{l}\text { Hospitalization for cardiovascular reasons, } n \\
\qquad(\%)\end{array}$ & $5(5.9 \%)$ & $5(8.5 \%)$ & 0.72 \\
\hline Death, $n(\%)$ & $1(1.2 \%)$ & $0(0 \%)$ & 1.0000 \\
\hline Death/hospitalization/stroke, $n(\%)$ & $6(7 \%)$ & $5(8.5 \%)$ & 0.742 \\
\hline PISA-difference from baseline (mm) & $-2.3(-6.0-+2.0)$ & $-4.9(-9.0-+1.0)$ & $<0.0001$ \\
\hline ERO-difference from baseline $\left(\mathrm{mm}^{2}\right)$ & $-6.7(-25-+12)$ & $-16.8(-45-+5)$ & $<0.0001$ \\
\hline MRvol-difference from baseline (mL) & $-9.4(-37-+28)$ & $-25.9(-57--3)$ & $<0.0001$ \\
\hline
\end{tabular}

Data are presented as median values and interquartile ranges, or number (percentage) as shown. PISA-proximal isovelocity surface area; ERO_effective regurgitant orifice; MRvol-mitral regurgitation volume.

We compared patients with at least moderate IMR after CABGa to patients without or only mild regurgitation one year after surgery (Table 6). To explore the effect of revascularization on the risk of recurrence of IMR in CABGa group, we analyzed changes in WMSI, which were stratified according to the recurrence of IMR defined as an ERO $>10 \mathrm{~mm}^{2}$ in 1-year follow-up. The improvement in the global WMSI was significantly higher for patients who were free of moderate or severe IMR at 1 year than for those with such mitral regurgitation (1.3 vs. 1.6 , respectively, $p=0.0109$ ). In the CABGa group, patients who never had recurrence of moderate or severe IMR and who had not undergone a mitral-valve intervention had lower LV volumes and EF than those with recurrence of IMR (ESV, 56.1 and $83.5 \mathrm{~mL}$, respectively; $p=0.002 ; \mathrm{EF}, 51.2$ and $42.6 \%$, respectively, $p=0.0005)$. The comparison of clinical and echocardiographic data in patients with IMR with ERO $\leq 10 \mathrm{~mm}^{2}$ and ERO $>10 \mathrm{~mm}^{2}$ in 1-year follow-up is presented in Table 6.

Table 6. Comparison of patients in CABGa group with IMR with ERO $\leq 10 \mathrm{~mm}^{2}$ and ERO $>10 \mathrm{~mm}^{2}$ in 1 year follow-up.

\begin{tabular}{|c|c|c|c|}
\hline & $\begin{array}{c}\text { ERO } \leq 10 \mathrm{~mm}^{2} \\
n=62(75 \%)\end{array}$ & $\begin{array}{c}\text { ERO > } 10 \mathrm{~mm}^{2} \\
n=28(25 \%)\end{array}$ & $p$ Value \\
\hline Male sex, $n(\%)$ & $35(56.5 \%)$ & $16(57.1 \%)$ & 1.0000 \\
\hline BMI $\left(\mathrm{kg} / \mathrm{m}^{2}\right)$ & $27.1(17.6-37.5)$ & $25.9(18-31.6)$ & 0.2356 \\
\hline Current smoking, $n(\%)$ & $6(9.7 \%)$ & $9(33.3 \%)$ & 0.0163 \\
\hline Hypertension, $n(\%)$ & $41(66.1 \%)$ & $17(61.9 \%)$ & 0.7936 \\
\hline Diabetes, $n(\%)$ & $43(69.4 \%)$ & $8(28.6 \%)$ & 1.0000 \\
\hline Hyperlipidemia, $n(\%)$ & $43(69.4 \%)$ & $13(47.6 \%)$ & 0.1136 \\
\hline Atrial fibrillation, $n(\%)$ & $10(16.1 \%)$ & $5(19.1 \%)$ & 0.7444 \\
\hline COPD, $n(\%)$ & $4(6.5 \%)$ & $4(14.3 \%)$ & 0.3618 \\
\hline NYHA class & $1.2(0-3)$ & $1.5(1-3)$ & 0.0336 \\
\hline CCS class & $1.0(1-2)$ & $1.1(1-2)$ & 0.0201 \\
\hline
\end{tabular}


Table 6. Cont.

\begin{tabular}{|c|c|c|c|}
\hline & $\begin{array}{c}\mathrm{ERO} \leq 10 \mathrm{~mm}^{2} \\
n=62(75 \%)\end{array}$ & $\begin{array}{c}\text { ERO > } 10 \mathrm{~mm}^{2} \\
n=28(25 \%)\end{array}$ & $p$ Value \\
\hline \multicolumn{4}{|l|}{ Medications, $n(\%)$ : } \\
\hline Beta-adrenolytics & $59(95.2 \%)$ & $27(95.2 \%)$ & 1.0000 \\
\hline ACE-I & $51(82.3 \%)$ & $24(85.7 \%)$ & 1.0000 \\
\hline CC blockers & $12(19.4 \%)$ & $1(4.8 \%)$ & 0.1683 \\
\hline Loop diuretics & $50(80.7 \%)$ & $24(85.7 \%)$ & 0.7500 \\
\hline Statins & $59(95.2 \%)$ & $25(90.5 \%)$ & 0.5965 \\
\hline ASA & $61((98.4 \%)$ & $8(90.5 \%)$ & 0.1562 \\
\hline LVDD (mm) & $50.5(41-62)$ & $54.5(39-68)$ & 0.0342 \\
\hline LVDS (mm) & $35.9(22-51)$ & $41.2(26-58)$ & 0.0062 \\
\hline EDV (mL) & $105.8(61-220)$ & $132.5(48-244)$ & 0.0634 \\
\hline ESV (mL) & $56.1(21-150)$ & $83.5(20-188)$ & 0.0223 \\
\hline $\mathrm{LA}(\mathrm{mm})$ & $40.3(30-54)$ & $45.7(35-65)$ & 0.0042 \\
\hline $\mathrm{EF}(\%)$ & $52.2(28-70)$ & $42.6(25-60)$ & 0.0005 \\
\hline WMSI & $1.3(1-1.94)$ & $1.6(1.1-2.5)$ & 0.0109 \\
\hline Workload (Watts) & $92.7(25-125)$ & $76.1(25-125)$ & 0.0109 \\
\hline
\end{tabular}

Data are presented as median values and interquartile ranges or number (percentage) as shown; CAD, coronary artery disease; BMI, body mass index; COPD, chronic obstructive pulmonary disease; CCS, Canadian Cardiovascular Society; NYHA, New York Heart Association; ACE-I, angiotensin converting enzyme inhibitors; CC blockers, calcium channel blockers; ASA, acetylsalicylic acid; WMSI, wall motion score index; EF, ejection fraction; LVDD, left ventricular end-diastolic dimension; LVDS, left ventricular end-systolic dimension; LA, left atrium; EDV, left ventricular end-diastolic volumes; ESV, left ventricular end-systolic volume.

Univariate analysis results with respect to the recurrence of moderate or severe IMR in the CABGa group are shown in Table 7. Left atrial dimensions and MDI's changes (TA and $\mathrm{CH}$ ) during DBX before surgery were significant predictors of the presence of at least moderate IMR in the CABGa group in a 12-month follow-up. Preoperative TAdbx and $\mathrm{CHdbx}$ were the independent predictors of the risk of recurrence of IMR. TAdbx $>1 \mathrm{~cm}^{2}$ provided a sensitivity of $90 \%$ and specificity of $29 \%$ (AUC 0.6436). The best cut-off value for CHdbx was $0.4 \mathrm{~cm}$ (sensitivity 90\%, specificity 34\%; AUC 0.6432) (Figures 2 and 3). None of the exercise echo parameters predicted the recurrence of IMR in a 12-month follow-up.

Table 7. Logistic regression analysis of predictors of ERO $>10 \mathrm{~mm} 2$ in CABGa group.

\begin{tabular}{|c|c|c|c|}
\hline \multirow{2}{*}{ Variable } & \multicolumn{3}{|c|}{ Univariate Analysis } \\
\hline & OR & $95 \%$ CI & $p$ Value \\
\hline Female sex & 1.029 & $0.379-2.794$ & 0.9560 \\
\hline BMI & 920 & $0.802-1.055$ & 0.2349 \\
\hline Smoking & 4.667 & $1.354-16.090$ & 0.0147 \\
\hline Hypertension & 0.832 & $0.298-2.322$ & 0.7258 \\
\hline Diabetes & 0.840 & $0.283-2.489$ & 0.7531 \\
\hline Hyperlipidemia & 0.402 & $0.146-1.105$ & 0.0774 \\
\hline Chronic kidney disease & 0.711 & $0.139-3.646$ & 0.6822 \\
\hline Atrial fibrillation & 1.224 & $0.339-4.411$ & 0.7578 \\
\hline COPD & 2.417 & $0.494-11.822$ & 0.2760 \\
\hline One vessel disease & 3.050 & $0.182-51.039$ & 0.4379 \\
\hline
\end{tabular}


Table 7. Cont.

\begin{tabular}{|c|c|c|c|}
\hline \multirow{2}{*}{ Variable } & \multicolumn{3}{|c|}{ Univariate Analysis } \\
\hline & OR & $95 \%$ CI & $p$ Value \\
\hline Two vessels disease & 0.441 & $0.115-1.691$ & 0.2326 \\
\hline Three vessels disease & 0.889 & $0.294-2.685$ & 0.8346 \\
\hline Beta-adrenolytic at discharge & 1.017 & $0.100-10.340$ & 0.9887 \\
\hline ACEI at discharge & 1.294 & $0.324-5.170$ & 0.7153 \\
\hline CC blockers at discharge & 0.208 & $0.025-1.710$ & 0.1441 \\
\hline Loop diuretics at discharge & 1.440 & $0.364-5.695$ & 0.6034 \\
\hline Statins at discharge & 0.483 & $0.075-3.110$ & 0.4438 \\
\hline ASA at discharge & 0.156 & $0.013-1.814$ & 0.1377 \\
\hline \multicolumn{4}{|l|}{ Rest TTE before surgery } \\
\hline $\mathrm{EF}$ & 0.960 & $0.960-1.014$ & 0.1468 \\
\hline WMSI & 2.330 & $0.504-10.774$ & 0.2790 \\
\hline LVDD & 1.039 & $0.961-1.123$ & 0.3351 \\
\hline LVDS & 1.027 & $0.963-1.095$ & 0.4200 \\
\hline EDV & 1.006 & $0.996-1.015$ & 0.2315 \\
\hline ESV & 1.007 & 0.996-1.018 & 0.2310 \\
\hline LA & 1.107 & $1.001-1.225$ & 0.0475 \\
\hline TRPG & 1.057 & $0.992-1.126$ & 0.0855 \\
\hline VC & 0.700 & $0.337-1.451$ & 0.3372 \\
\hline PISA & 1.297 & $0.804-2.093$ & 0.2871 \\
\hline ERO & 1.33 & $0.008-18.45$ & 0.1876 \\
\hline MR volume & 1.077 & $0.998-1.163$ & 0.0573 \\
\hline SIs & 30.790 & $0.172-68.23$ & 0.1952 \\
\hline SId & 4.253 & $0.051-351.768$ & 0.547 \\
\hline Coaptation height & 6.262 & $0.436-89.843$ & 0.1770 \\
\hline Tenting area & 2.955 & $0.958-9.113$ & 0.0593 \\
\hline \multicolumn{4}{|l|}{ Dobutamine TTE before surgery } \\
\hline $\mathrm{EF}$ & 0.961 & 0.913-1.012 & 0.1337 \\
\hline WMSI & 4.384 & $0.727-26.455$ & 0.1070 \\
\hline PISA & 1.561 & $1.010-2.414$ & 0.0450 \\
\hline ERO & 1.23 & $0.039-23.1$ & 0.1115 \\
\hline MR volume & 1.103 & $0.993-1.225$ & 0.0669 \\
\hline Coaptation height (CHdbx) & 5.52 & $1.037-92.7$ & 0.0480 \\
\hline Tenting area (TAdbx) & 6.307 & $1.190-33.424$ & 0.0304 \\
\hline \multicolumn{4}{|c|}{ Exercise echocardiography before surgery } \\
\hline $\mathrm{EF}$ & 0.958 & $0.908-1.011$ & 0.1184 \\
\hline
\end{tabular}


Table 7. Cont.

\begin{tabular}{ccccc}
\hline \multirow{2}{*}{ Variable } & \multicolumn{3}{c}{ Univariate Analysis } \\
\cline { 2 - 5 } & OR & 95\% CI & $p$ Value \\
\hline TRPG & 1.040 & $0.996-1.086$ & 0.0757 & 0.1306 \\
\hline PISA & 1.243 & $0.938-1.647$ & 0.1447 \\
\hline
\end{tabular}

CAD, coronary artery disease; BMI, body mass index; COPD, chronic obstructive pulmonary disease; CCS, Canadian Cardiovascular Society; NYHA, New York Heart Association; ACE-I, angiotensin converting enzyme inhibitors; CC blockers, calcium channel blockers; ASA, acetylsalicylic acid; WMSI, wall motion score index; EF, ejection fraction; LVDD, left ventricular end-diastolic dimension; LVDS, left ventricular end-systolic dimension; LA, left atrium; EDV, left ventricular end-diastolic volumes; ESV, left ventricular end-systolic volume; rest, echo examination at rest; exe, exercise; dbx, dobutamine echocardiography; VC, vena contracta; PISA, proximal isovelocity surface area; ERO, effective regurgitant orifice; MRvol, mitral regurgitation volume; TA, tenting area; $\mathrm{CH}$, coaptation height; Sis, sphericity index at end-systole; Sis, sphericity index at end-diastole TRPG, maximal tricuspid regurgitant peak gradient; LA, left atrium; MR, mitral regurgitation.

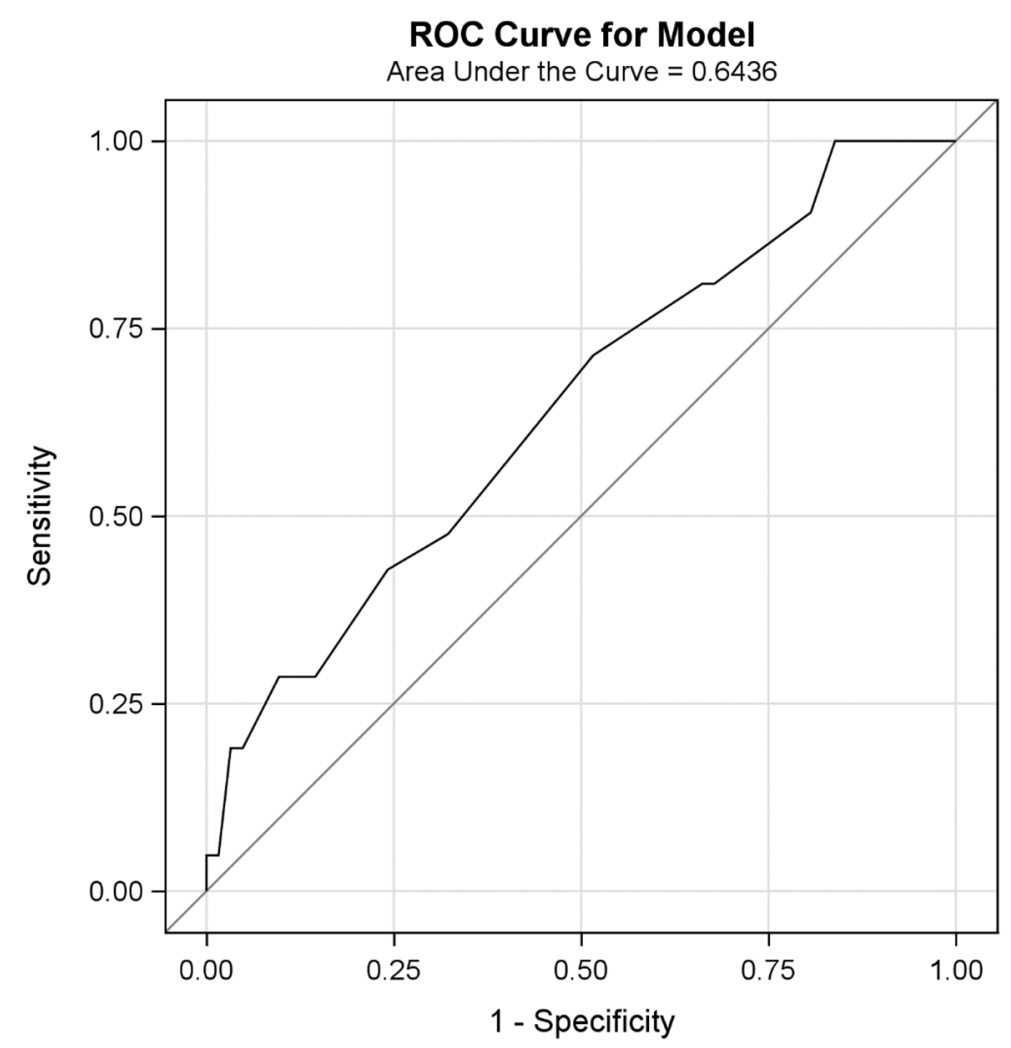

Figure 2. Receiver operator characteristic (ROC) curve for TA during DBX as a predictor of mitral regurgitation with $\mathrm{ERO}>10 \mathrm{~mm}^{2}$ in patients 12 month after CABG. 


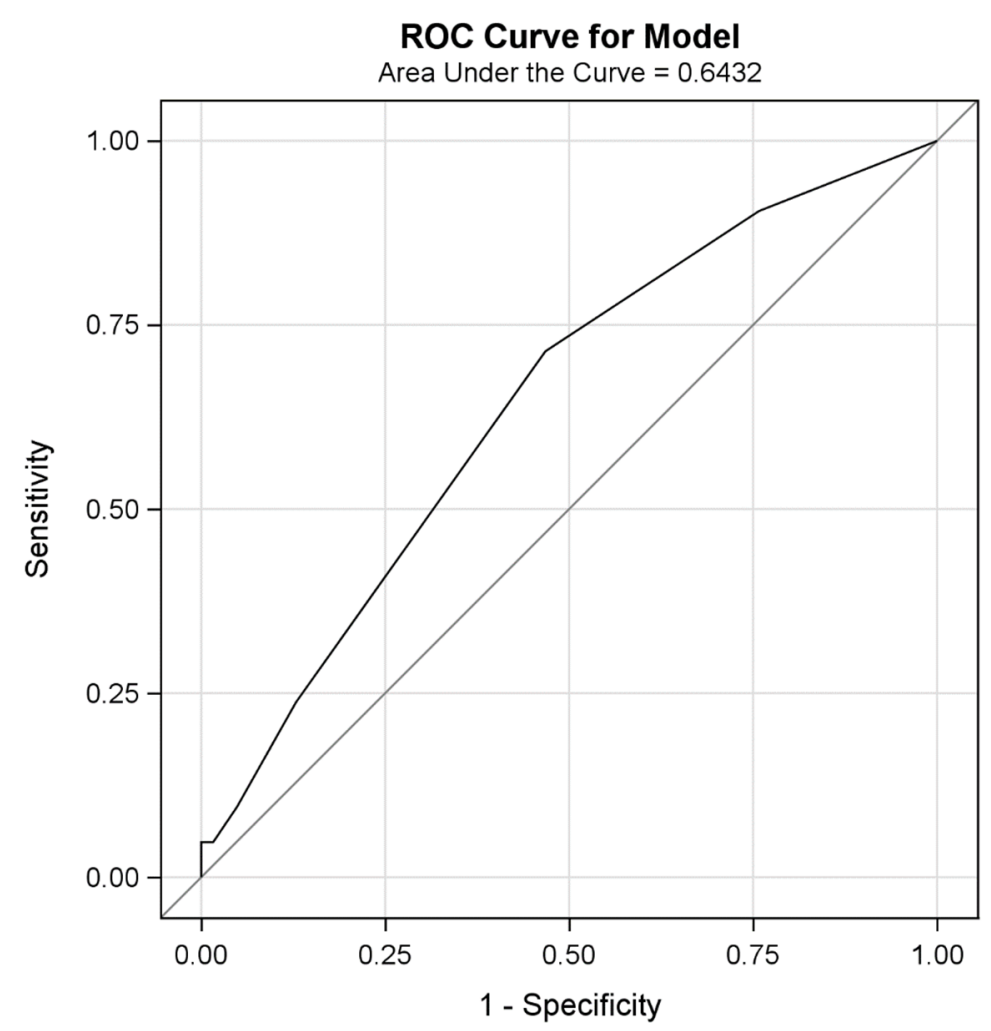

Figure 3. Receiver operator characteristic (ROC) curve for $\mathrm{CH}$ during $\mathrm{DBX}$ as a predictor of mitral regurgitation with ERO $>10 \mathrm{~mm}^{2}$ in patients 12 month after CABG.

\subsection{Clinical Outcomes}

At 1 year, we observed no significant difference in death rates between the study groups, with $1.2 \%$ for CABGa and $0 \%$ for the combined procedure $(p=1.000)$. Overall rates of cardiovascular rehospitalizations did not differ significantly in the two study groups (CABGa vs. CABGmp: $5.9 \%$ vs. $8.5 \%, p=0.72$ ). In addition, there were no significant differences in the composite endpoint of major adverse cardiac or cerebrovascular events (death/hospitalization/stroke) between the study groups (CABGa vs. CABGmp: 7\% vs. $8.5 \% ; p=0.742)$ (Table 5).

\section{Discussion}

In our study, the qualification of patients for CABG alone was based on very strict echocardiographic criteria, the crucial element of which was to reveal the myocardial viability of those LV segments, the dysfunction of which during rest examination generated IMR. A cumulative analysis of ExE and DBX results allowed for the final therapeutic decisions. Simultaneously, an indispensable condition was to obtain a complete normalization of MDI and a decrease in IMR grade during DBX. Roshanali et al. used only simple qualitative echocardiographic criteria based on the 4-grade scale of mitral regurgitation to identify patients with moderate IMR. They showed the utility of low-dose DBX in selecting patients who would be undergoing CABG to receive concurrent mitral valve repair $\{13\}$. We have developed a more advanced protocol based on exercise echocardiography (ExE) and dobutamine stress echocardiography (DBX) criteria for the precise determination of the range of surgical interventions. The presented criteria allowed us to qualify 90 patients for this type of treatment. Unfortunately, postoperative results showed that some patients in the CABGa group have at least moderate IMR in 1 year after surgery. The main aim of the study was to predict failure using preoperative information, and providing the clinician with guidance regarding the choice of therapy. In the CABGa group, a statistically significant IMR reduction was found in most of patients in follow-up. The complete revascularization performance resulted in significant improvement in LV geometry and function. A reduction 
in the degree of mitral regurgitation with CABG alone has been reported previously [27-29]. Penicka et al. found that in a series of patients with moderate IMR who underwent CABG alone, the resolution of MR after surgery was associated with more viable segments and less LV desynchrony at baseline [30]. Kang et al. reported that patients who demonstrated an improvement in LV function and a reduction in LV size after CABG also had a reduction in the IMR grade one year after surgery [28]. In our study, we observed a similar reduction in the IMR in the CABGa group. The progress of LV remodeling and, secondarily, the posterior mitral valve leaflet restriction, is a mechanism responsible for the lack of improvement or increase in the IMR grade after surgery [31]. In our study, we observed a decrease in LV volumes and diameters, as well as an increase in EF in most patients; favorable echocardiography results were reflected in the patient's clinical status. Moreover, the absence of IMR after surgery was associated with improvement in global wall-motion scores at 1 year. Michler et al. randomly assigned 301 patients with moderate IMR to undergo either $\mathrm{CABG}$ alone or the combined procedure with mitral repair. They reported that improvements in both global and regional wall motion scores and the presence of $\mathrm{LV}$ reverse remodeling were associated with significantly less moderate or severe mitral regurgitation at a 2 year follow-up [32]. Improvement in global wall motion score indexes, decreases in LV volumes, and increases in EF after revascularization are indicative of viable myocardium. As a result, it can also improve mitral valve function in patients with IMR in relation to the decrease in LV size, increased mitral valve closing forces, improved papillary-muscle synchrony, and enhanced myocardial contractility. Therefore, surgical decision making could be improved by identifying which patients are most likely to have an improvement in LV function after revascularization, which can lead to a postoperative reduction in IMR. In our study, in all patients, we assessed both myocardial viability and changes in mitral valve geometry (tenting area and coaptation heigh) using low-dose DBX and exercise echocardiography. We observed significant correlations between preoperative changes in MDI and the presence of significant IMR 1 year after surgery by using DBX, but not exercise echocardiography. These findings suggest that DBX-induced reversible ischemia changes, especially in the posterior wall, could improve MDI. Improvement in wall motion contractility in segments supporting the posterior papillary muscle may reduce the degree of IMR by decreasing leaflet tethering forces that cause incomplete mitral leaflet closure. Abe et al. reported similar observations in a small group of patients with coronary artery disease and moderate IMR [33]. Our observations also suggests that the presence of contractile reserve, as well as improvement in MDIs, identified with DBX, may serve as a predictor of reduced IMR in response to revascularization in patients qualified to CABG.

Despite the higher proportion of patients with moderate or severe IMR at 1 year in the CABGa group, 1-year clinical outcomes, including functional status, mortality, and major adverse cardiac and cerebrovascular events, did not differ significantly between the study groups. Our results are like those of one randomized trial involving patients with moderate ischemic mitral regurgitation [34]. However, our study was not powered to detect small, but important differences in survival and clinical composite endpoints.

The results of our study indicate that some patients experience a recurrence of IMR after surgery. Currently, in patients with a recurrence of significant IMR after CABGaor CABGmp (especially after failed surgical mitral valve repair), MitraClip may be a good option [35]. However, our study was conducted between 2010 and 2017, when MitraClip interventions were not as available as they are today.

To our knowledge, this is the first prospective case-series analysis of the patient's qualification for surgical treatment based on all essential elements of mitral complex functioning in echocardiography examination at rest, as well as during stress echo.

The main limitation of the study was a relatively low number of included patients. Therefore, the statistical power of correlations is lowered. Because of the low value of events per variable, multivariate models were not analyzed. It must be stressed, however, that significant IMR in patients treated with CABGa is seen only in a small number of patients. Taking into consideration the innovative character of this study, the collected 
group of patients belongs to the most numerous presented in the literature, and is the first with such a complex methodology of a patient's qualification.

Another limitation of the study is the lack of randomization, which may result in potential selection bias, possibly leading to incorrect conclusions. It must be stressed that in the present study, deterministic criteria for patients' qualification to the suitable type of treatment were used. A lack of randomization of patients resulted in uneven selection to the CABGa and CABGmp groups.

Our study results indicate that the application of an elaborated diagnostic algorithm may improve the qualification of patients with significant IMR for a suitable type of surgical procedure, finally enabling good clinical results in the 12-month follow-up after surgery. Based on such qualification in selected patients, the CABG alone can ameliorate IMR and produce beneficial functional and structural improvements without an increase in long-term mortality. The current guidelines emphasize that in patients with severe IMR, valve surgery is recommended in patients undergoing CABG. In selected patients without advanced LV remodeling, mitral valve repair is recommended. In contrast, additional valve replacement may be considered in patients with echocardiographic predictors of repair failure.

The guidelines also emphasize the importance of exercise echocardiography, which may help to identify patients with severe mitral regurgitation when echocardiography at rest is inconclusive [36]. Our study included the use of exercise echocardiography in the qualification protocol to evaluate changes in IMR during an exercise. The guidelines highlight the difficulties in managing patients with moderate IMR. They emphasize that surgery is more likely to be considered if myocardial viability is present, and if comorbidity is low. In addition, an exercise-induced large increase in mitral regurgitation severity and systolic pulmonary artery pressure favors combined surgery (CABG with mitral intervention) [35]. Our study protocol included an assessment of all these elements (myocardial viability and exercise induced IMR changes). In addition, it included the assessment of changes in the geometry of the mitral valve during DBX, which is important in the pathomechanism of IMR.

Because this novel clinical decision-making tool has been applied in a small group of patients, the present results must be considered hypothesis generating.

This must be clearly stated, along with the need to study a larger group before broadly implementing the approach. Certainly, further validation and randomized studies with more patients enrolled and followed-up for a longer period are necessary.

In conclusion, it should be emphasized that CABG alone could be contemplated if IMR parameters improve with preoperative DBX, and do not impair with EXE. Moreover, CABGmp could be considered for patients without IMR improvement during DBX, and with deterioration during ExE. That decision should be individualized for patients with just one stress test (DBX or EXE) showing positive findings.

This study has highlighted the importance of detailed preoperative TTE examination (rest and stress echo) in patients undergoing surgery for IMR. It can be used to identify patients with IMR who are likely to have recurrent IMR after CABG. Specifically, higher LV volumes, lower EF, higher tethering area at rest TTE, and changes of MDI indexes during preoperative DBX were associated with a recurrence of IMR. In these patients, changes and additional repair techniques or MV replacement should be considered.

Author Contributions: Conceptualization, R.P., J.K., and G.O.; software, M.P., M.B., and R.P.; validation, R.P., M.P., and M.B.; data curation, J.K., R.P., and M.G.; writing-original draft preparation, R.P., M.B., M.P., and J.K.; writing-final review and editing, R.P., M.B., M.P., and G.O. All authors have read and agreed to the published version of the manuscript.

Funding: This research received no external funding.

Institutional Review Board Statement: The study was approved by the institutional review board of the Medical University of Warsaw. The study had been conducted according to the principles stated in the Declaration of Helsinki. 
Informed Consent Statement: Each patient signed an informed consent form.

Data Availability Statement: Additional data are available upon request from the first author (R.P.).

Conflicts of Interest: The authors declare no conflict of interest.

\section{References}

1. Lamas, G.A.; Mitchell, G.F.; Flaker, G.C.; Smith, S.C.; Gersh, B.J.; Basta, L.; Moye, L.; Braunwald, E.; Pfeffer, M.A. Clinical significance of mitral regurgitation after acute myocardial infarction. Surviv. Ventricular Enlarg. Investig. Circ. 1997, 96, 827-833. [CrossRef] [PubMed]

2. Grigioni, F.; Enriquez-Sarano, M.; Zehr, K.; Bailey, K.; Tajik, A. Ischemic mitral regurgitation. Long-term outcome and prognostic implications with quantitative Doppler assessment. ACC Curr. J. Rev. 2001, 10, 33. [CrossRef]

3. Grigioni, F.; Detaint, D.; Avierinos, J.-F.; Scott, C.; Tajik, J.; Enriquez-Sarano, M. Contribution of ischemic mitral regurgitation to congestive heart failure after myocardial infarction. J. Am. Coll. Cardiol. 2005, 45, 260-267. [CrossRef]

4. Kwan, J.; Shiota, T.; Agler, D.A.; Popović, Z.B.; Qin, J.X.; Gillinov, M.A.; Stewart, W.J.; Cosgrove, D.M.; McCarthy, P.M.; Thomas, J.D. Geo-metric differences of the mitral apparatus between ischemic and dilated cardiomyopathy with significant mitral regurgitation: Real-time three-dimensional echocardiography study. Circulation 2003, 107, 1135-1140. [CrossRef]

5. Zhu, F.; Otsuji, Y.; Yotsumoto, G.; Yuasa, T.; Ueno, T.; Yu, B.; Koriyama, C.; Hamasaki, S.; Biro, S.; Kisanuki, A.; et al. Mechanism of persistent ischemic mitral regurgitation after annuloplasty: Importance of augmented posterior mitral leaflet tethering. Circulation 2005, 30, I396-I401.

6. Agricola, E.; Oppizzi, M.; Maisano, F.; De Bonis, M.; Schinkel, A.F.; Torracca, L.; Margonato, A.; Melisurgo, G.; Alfieri, O. Echocardiographic classification of chronic ischemic mitral regurgitation caused by restricted motion according to tethering pattern. Eur. J. Echocardiogr. 2004, 5, 326-334. [CrossRef]

7. Watanabe, N.; Ogasawara, Y.; Yamaura, Y.; Yamamoto, K.; Wada, N.; Kawamoto, T.; Toyota, E.; Akasaka, T.; Yoshida, K. Geometric Differences of the Mitral Valve Tenting Between Anterior and Inferior Myocardial Infarction with Significant Ischemic Mitral Regurgitation: Quantitation by Novel Software System with Transthoracic Real-time Three-dimensional Echocardiography. J. Am. Soc. Echocardiogr. 2006, 19, 71-75. [CrossRef] [PubMed]

8. Srichai, M.B.; Grimm, R.A.; Stillman, A.E.; Gillinov, A.M.; Rodriguez, L.L.; Lieber, M.L.; Lara, A.; Weaver, J.A.; McCarthy, P.M.; White, R.D. Ischemic mitral regurgitation: Impact of LV and MV in patients with left ventricular systolic dysfunction. Ann. Thorac. Surg. 2005, 80, 170-178. [CrossRef]

9. Mihaljevic, T.; Lam, B.-K.; Rajeswaran, J.; Takagaki, M.; Lauer, M.; Gillinov, A.M.; Blackstone, E.H.; Lytle, B.W. Impact of Mitral Valve Annuloplasty Combined with Revascularization in Patients With Functional Ischemic Mitral Regurgitation. J. Am. Coll. Cardiol. 2007, 49, 2191-2201. [CrossRef]

10. Diodato, M.D.; Moon, M.R.; Pasque, M.K.; Barner, H.B.; Moazami, N.; Lawton, J.S.; Bailey, M.S.; Guthrie, T.J.; Meyers, B.F.; Damiano, R.J. Repair of ischemic mitral regurgitation does not increase mortality or improve long-term survival in patients undergoing coronary artery revascularization: A propensity analysis. Ann. Thorac. Surg. 2004, 78, 794-799. [CrossRef] [PubMed]

11. Lancellotti, P.; Troisfontaines, P.; Toussaint, A.C.; Pierard, L.A. Prognostic importance of exercise-induced changes in mitral regur-gitation in patients with chronic ischemic left ventricular dysfunction. Circulation 2003, 108, 1713-1717. [CrossRef] [PubMed]

12. Lancellotti, P.; Gerard, P.L.; Pierard, L.A. Long-term outcome of patients with heart failure and dynamic functional mitral regur-gitation. Eur. Heart J. 2005, 26, 1528-1532. [CrossRef] [PubMed]

13. Roshanali, F.; Mandegar, M.H.; Yousefnia, M.A.; Alaeddini, F.; Wann, S. Low-Dose Dobutamine Stress Echocardiography to Predict Reversibility of Mitral Regurgitation with CABG. Echocardiography 2006, 23, 31-37. [CrossRef]

14. Peteiro, J.; Bendayan, I.; Mariñas, J.; Campos, R.; Bouzas, B.; Castro-Beiras, A. Prognostic value of mitral regurgitation assessment during exercise echocardiography in patients with left ventricular dysfunction: A follow-up study of $1.7 \pm 1.5$ years? Eur. J. Echocardiogr. 2007, 9, 18-25. [CrossRef]

15. HHillis, L.D.; Smith, P.K.; Bittl, J.A.; Bridges, C.R.; Byrne, J.G.; Cigarroa, J.E.; DiSesa, V.J.; Hiratzka, L.F.; Hutter, A.M.; Jessen, M.E.; et al. 2011 ACCF/AHA guideline for coronary artery bypass graft surgery a report of the American College of Cardi-ology Foundation/American Heart Association Task Force on Practice Guidelines. Circulation 2001, 124, e652-e735.

16. Braun, J.; Voigt, P.G.; Holman, E.R.; Bax, J.J.; Versteegh, M.I.; Klautz, R.J.; Boersma, E.; Dion, R.A. Preoperative left ventricular dimensions predict reverse remodeling following restrictive mitral annuloplasty in ischemic mitral regurgitation? Eur. J. Cardio-Thorac. Surg. 2005, 27, 847-853. [CrossRef] [PubMed]

17. Spoor, M.T.; Geltz, A.; Bolling, S.F. Flexible Versus Nonflexible Mitral Valve Rings for Congestive Heart Failure: Differential Durability of Repair. Circulation 2006, 114, I67. [CrossRef]

18. Baumgartner, H.; Falk, V.; Bax, J.J.; De Bonis, M.; Hamm, C.; Holm, P.J.; Iung, B.; Lancellotti, P.; Lansac, E.; Rodriguez Muñoz, D.; et al. 2017 ESC/EACTS Guidelines for the management of valvular heart disease. Eur. Heart J. 2017, 38, 2739-2791. [CrossRef]

19. Piérard, L.A.; Carabello, B.A. Ischaemic mitral regurgitation: Pathophysiology, outcomes, and the conundrum of treatment. Eur. Hear. J. 2010, 31, 2996-3005. [CrossRef]

20. Lancellotti, P.; Marwick, T.A.; Pierard, L. How to manage ischaemic mitral regurgitation. Heart 2008, 94, 1497-1502. [CrossRef]

21. Enriquez-Sarano, M.; Bailey, K.R.; Seward, J.B.; Tajik, A.J.; Krohn, M.J.; Mays, J.M. Quantitative Doppler assessment of valvular regurgitation. Circulation 1993, 87, 841-848. [CrossRef] [PubMed] 
22. Vahanian, A.; Baumgartner, H.; Bax, J.; Butchart, E.; Dion, R.; Filippatos, G.; Flachskampf, F.; Hall, R.; Iung, B.; Kasprzak, J.; et al. Guidelines on the management of valvular heart disease: The Task Force on the Management of Valvular Heart Disease of the European Society of Cardiology. Eur. Hear. J. 2006, 28, 230-268. [CrossRef]

23. Cerqueira, M.D.; Weissman, N.J.; Dilsizian, V.; Jacobs, A.K.; Kaul, S.; Laskey, W.K.; Pennell, D.J.; Rumberger, J.A.; Ryan, T.; Verani, M.S. Standardized myocardial segmentation and nomenclature for tomographic imaging of the heart: A statement for healthcare professionals from the cardiac imaging committee of the council on clinical cardiology of the american heart association. Circulation 2002, 105, 539-542. [PubMed]

24. Yiu, S.F.; Enriquez-Sarano, M.; Tribouilloy, C.; Seward, J.B.; Tajik, A.J. Determinants of the degree of functional mitral regurgitation in patients with systolic left ventricular dysfunction: A quantitative clinical study. Circulation 2000, 102, 1400-1406. [CrossRef]

25. Bax, J.J.; Poldermans, D.; Elhendy, A.; Cornel, J.H.; Boersma, E.; Rambaldi, R.; Roelandt, J.R.; Fioretti, P.M. Improvement of left ventricular ejection fraction, heart failure symptoms and prognosis after revascularization in patients with chronic coronary artery disease and viable myocardium detected by dobutamine stress echocardiography. J. Am. Coll. Cardiol. 1999, 34, 163-169. [CrossRef]

26. Pellikka, P.A.; Nagueh, S.F.; Elhendy, A.A.; Kuehl, C.A.; Sawada, S.G. American Society of Echocardiography Recommendations for Performance, Interpretation, and Application of Stress Echocardiography. J. Am. Soc. Echocardiogr. 2007, 20, 1021-1041. [CrossRef]

27. Mallidi, H.R.; Pelletier, M.P.; Lamb, J.; Desai, N.; Sever, J.; Christakis, G.T.; Cohen, G.; Goldman, B.S.E.; Fremes, S. Late outcomes in patients with uncorrected mild to moderate mitral regurgitation at the time of isolated coronary artery bypass grafting. $J$. Thorac. Cardiovasc. Surg. 2004, 127, 636-644. [CrossRef]

28. Kang, D.H.; Kim, M.J.; Kang, S.J.; Song, J.M.; Song, H.; Hong, M.K.; Choi, K.J.; Song, J.K.; Lee, J.W. Mitral valve repair versus revascularization alone in the treatment of ischemic mitral re-gurgitation. Circulation 2006, 114, I-499-I-503. [CrossRef]

29. Rydén, T.; Bech-Hanssen, O.; Brandrup-Wognsen, G.; Nilsson, F.; Svensson, S.; Jeppsson, A. The importance of grade 2 ischemic mitral regurgitation in coronary artery bypass grafting. Eur. J. Cardio-Thorac. Surg. 2001, 20, 276-281. [CrossRef]

30. Penicka, M.; Linkova, H.; Lang, O.; Fojt, R.; Kočka, V.; Vanderheyden, M.; Bartunek, J. Predictors of Improvement of Unrepaired Moderate Ischemic Mitral Regurgitation in Patients Undergoing Elective Isolated Coronary Artery Bypass Graft Surgery. Circulation 2009, 120, 1474-1481. [CrossRef]

31. Hung, J.; Papakostas, L.; Tahta, S.A.; Hardy, B.G.; Bollen, B.A.; Duran, C.M.; Levine, R.A. Mechanism of recurrent ischemic mitral re-gurgitation after annuloplasty: Continued lv remodeling as a moving target. Circulation 2004, 110, II85-II90. [CrossRef] [PubMed]

32. Michler, R.E.; Smith, P.K.; Parides, M.K.; Ailawadi, G.; Thourani, V.; Moskowitz, A.J.; Acker, M.A.; Hung, J.W.; Chang, H.L.; Perrault, L.P.; et al. Two-Year Outcomes of Surgical Treatment of Moderate Ischemic Mitral Regurgitation. N. Engl. J. Med. 2016, 374, 1932-1941. [CrossRef] [PubMed]

33. Abe, Y.; Imai, T.; Ohue, K.; Otsuka, R.; Komatsu, R.; Otsuka, M.; Sakanoue, Y.; Naruko, T.; Itoh, A.; Yoshiyama, M.; et al. Relation between reduction in ischaemic mitral regurgitation and improvement in regional left ventricular contractility during low dose dobutamine stress echocardiography. Heart. 2005, 91, 1092-1093. [CrossRef] [PubMed]

34. Smith, P.K.; Puskas, J.D.; Ascheim, D.D.; Voisine, P.; Gelijns, A.C.; Moskowitz, A.J. Cardiothoracic Surgical Trials Network In-vestigators. Surgical treatment of moderate ischemic mitral regurgitation. N. Engl. J. Med. 2014, 371, 2178-2188. [CrossRef] [PubMed]

35. Rahhab, Z.; Lim, D.S.; Little, S.H.; Taramasso, M.; Kuwata, S.; Saccocci, M.; Tamburino, C.; Grasso, C.; Frerker, C.; Wißt, T.; et al. MitraClip After Failed Surgical Mitral Valve Repair-An International Multicenter Study. J. Am. Heart. Assoc. 2021, 10 , e019236. [CrossRef] [PubMed]

36. Vahanian, A.; Beyersdorf, F.; Praz, F.; Milojevic, M.; Baldus, S.; Bauersachs, J.; Capodanno, D.; Conradi, L.; De Bonis, M.; De Paulis, R.; et al. 2021 ESC/EACTS Guidelines for the management of valvular heart disease: Developed by the Task Force for the management of valvular heart disease of the European Society of Cardiology (ESC) and the European Association for Cardio-Thoracic Surgery (EACTS). Eur. Heart J. 2021, ehab395. [CrossRef] 